\title{
Novel Technique of Auto Transplantation of Ectopic Canines in Growing Patients
}

\author{
Paweł Plakwicz*1, Ewa Monika Czochrowska ${ }^{2}$ and Krzysztof Kukuła ${ }^{3}$ \\ ${ }^{1}$ Department of Periodontology, Medical University of Warsaw ul Miodowa, Poland \\ ${ }^{2}$ Department of Orthodontics, Medical University of Warsaw ul Miodowa, Poland \\ ${ }^{3}$ Department of Oral surgery, Medical University of Warsaw ul Miodowa, Poland
}

Received: February 02, 2018; Published: February 15, 2018

*Corresponding author: Paweł Plakwicz, Associate Professor, Department of Periodontology, Medical University of Warsaw ul. Miodowa 18, 00-443 Warsaw, Poland, Email: info@plakwicz.com

\begin{abstract}
Ectopic teeth can be auto transplanted to normal position under certain conditions such as orthodontic and surgical indications and optimal healing of transplants' tissues after surgery. The healing depends on of extent of osteotomy and amount of trauma to the root and periodontal ligament during the surgery. The aim of this paper is to introduce an innovative technique, which reduces trauma during auto transplantation thus allowing uncomplicated healing. A mandibular ectopic developing canine was diagnosed in 11-year-old girl. There were orthodontic contraindications for extraction of the affected tooth. Success of treatment would have been thus dependant on the amount of trauma received during surgery and normal healing after transplantation to normal position. For that reason the novel technique for use of orthodontic bracket was applied to limit trauma to the root during removal of the tooth. The canine's crown was exposed, orthodontic bracket was bonded to the enamel and wire was attached to the bracket. The pulling force were applied to the bracket and allowed controlled and gentle removal of canine from initial intraosseous position without abrading its root against surrounding bone. The released canine was immediately transplanted to normal position. One year after surgery the canine erupted spontaneously and presented normal mobility and healthy soft and hard tissues. The use of orthodontic bracket may contribute to reduce trauma to periodontal tissues during removal of impacted teeth for auto transplantation thus promoting uncomplicated healing after surgery.
\end{abstract}

Keywords: Healing outcome; Impacted canine; Surgical techniques; Tooth auto transplantation; Tooth ectopia

\section{Introduction}

Ectopic developing teeth in growing patients usually require surgical intervention. Treatment of affected tooth depends on age, orthodontic conditions, and severity of impaction and includes surgical exposure of affected tooth followed by orthodontic extrusion, auto transplantation, or extraction [1-4]. If auto transplantation of ectopic tooth is scheduled, the gentlest surgical technique of tooth removal is obligatory to achieve uncomplicated healing of periodontal tissues after surgery [5-7]. Gentle removal of the impacted tooth from its initial position within the bone crypt is challenging, usually requires osteotomy and use of the elevators and/or forceps. Application of conventional techniques and instrumentation may therefore damage the root surface of the transplant and impede optimal healing leading to complications such as replacement root resorption or restricted root development after surgery.

\section{Aim}

The aim of this paper is to describe the novel use of orthodontic bracket with attached steel wire to perform the gentlest removal of the ectopic lower canine from its intraosseous position. It was performed in order to avoid the risk of healing complications after transplantation of developing canine to its normal position.

\section{Case Description}

An 11-year-old girl attended for an orthodontic consultation at the University Hospital. Clinical examination revealed mixed dentition with retained primary canines. The radiographic examination revealed slightly horizontal position of unerupted permanent maxillary canines (\#13, \#23), rotation of unerupted mandibular right canine (\#43) and ectopic intraosseous position of mandibular left canine (\#33) which presented transmigration in the area of contralateral mandibular central incisor (Figure 1). All permanent canines had developing roots. There were orthodontic contraindications for extraction of ectopic canine (\#33) because of patient's occlusal conditions. The treatment plan included gentle removal of the transmigrated developing mandibular canine (\#33) from the bone and its immediate transplantation to optimal position. In such case, the success of treatment would directly 
depend on the healing of periodontal ligament after transplantation of affected canine. Solving other canines' position was scheduled after transplantation and healing of the transmigrated canine. Informed consent was obtained from patient and parents before the treatment.

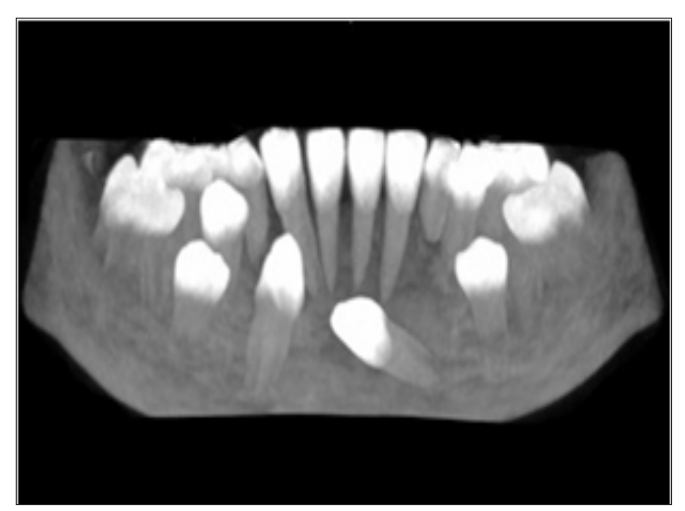

Figure 1: Maximum Intensity Projection scan (MIP) from CBCT taken before treatment showing the position of impacted developing tooth \#33 in relation to the mandibular incisors.



Figure 2: The surgery: the labial view after flap preparation and removal of buccal bone to expose crown of tooth \#33. The orthodontic bracket was bonded to the surface of the crown and the wire was attached to the bracket. Position of the Pean forceps indicates direction of pulling forces applied through the wire and bracket to the tooth (a); gentle extraction of the canine from bone crypt (b); extracted canine was placed in an artificially created root socket and stabilized with sutures in proper location (c); the flap was repositioned and sutured above transplanted canine $(\mathrm{d})$.

\section{Surgical Technique}

Under the local anesthesia (articaine with epinefrine 1: 200.000) the incision was made along the vestibular gingival margin and a full-thickness flap was created labially between right primary canine (\#75) and left first premolar in the mandible. The left primary canine was extracted. The labial cortical bone which covered ectopic left canine (\#33) was removed and the dental follicle was partially excised to expose the labial surface of the crown of impacted tooth. After selected etching and rinsing of the exposed enamel, the orthodontic bracket (Lingual button curved,
Dentsply GAC International Inc. USA) was bonded to the etched surface using light-cured flow composite resin (Figure 2a). The steel wire was attached to the previously bonded bracket. Pulling and slight lateral movements ware applied to the wire by Pean forceps to gently extrude the ectopic canine from its crypt within the bone (Figure 2b). The osteotomy to create artificial socket with use of surgical burs was performed at the site corresponding to normal position of mandibular left canine after removal of the primary canine. During preparation of the socket, the permanent canine was kept in saline solution for about four minutes. The canine was than transplanted to the artificially created socket. The new socket was partially missing labial wall because the osteotomy was performed within narrow alveolus that could not accommodate the entire width of the canine. Due to this fact, the tooth was positioned slightly labially and stabilized with resorbable sutures (Figure 2c). The mucogingival flap was sutured to completely cover transplanted tooth and bone dehiscence on its root (Figure $2 \mathrm{~d}$ ). The intra-oral radiograph was taken at the end of surgery to confirm adequate position of the canine (Figure 3a).

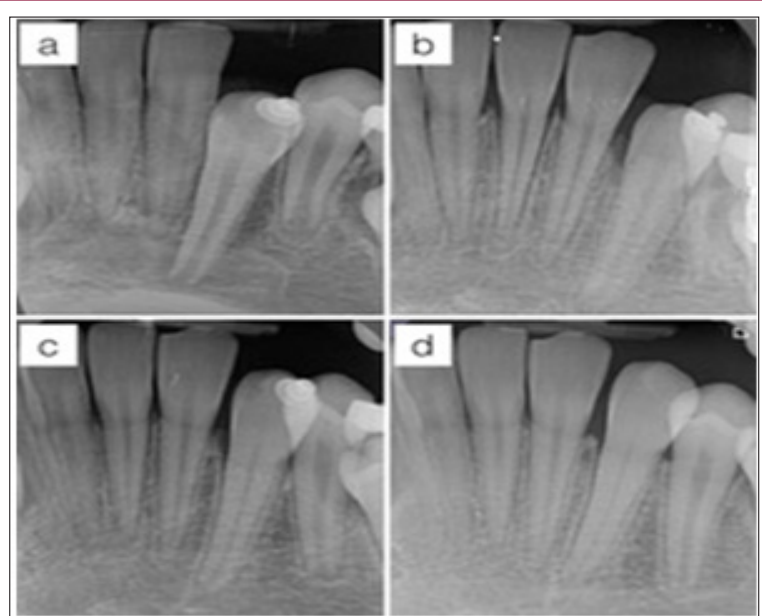

Figure 3: Radiographic examination (intra-oral X-rays) of transplanted canine in different periods after transplantation: immediately after surgery (a); four months follow-up (b); eight months follow-up (c) showing root growth and spontaneous eruption (c). 12 months radiographic follow-up showed complete eruption, partial pulp obliteration and absence of pathology related to bone and tooth (d).

\section{Follow-up and final examination}

The healing was uncomplicated with no signs of edema or inflammation and sutures were removed 14 days after surgery. Four months later the transplanted canine spontaneously erupted through the gingiva (Figure 4a). Another four months later the orthodontic bracket was debonded and the enamel has been cleaned of composite residues (Figures $4 \mathrm{~b} \& 4 \mathrm{c}$ ). One year after transplantation the canine fully erupted to the contact with opposite arch (Figure 4d), showed normal mobility and responded to vitality tests within normal limits. The periodontal tissues were healthy (probing depth was less than $2 \mathrm{~mm}$, normal clinical attachment level and no bleeding on probing) with wide keratinized gingiva around the tooth. The intra-oral radiograph was taken at eight 
and 12 months follow-up (Figures 3b \& 3c). It revealed eruption and progressive pulp obliteration which were normal findings after transplantation of developing teeth. It also confirmed reestablishment of lamina dura of alveolar socket with no pathology related to bone or root of the transplant.

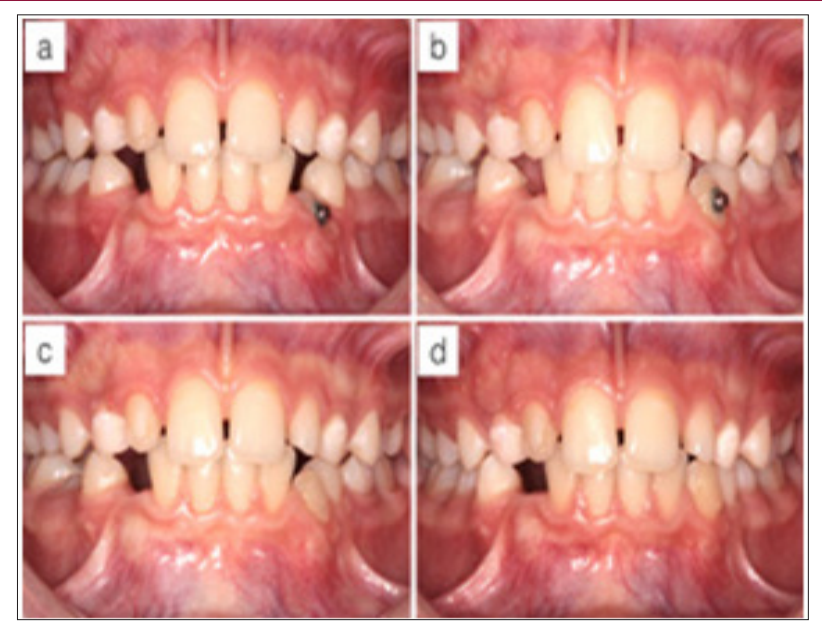

Figure 4: Clinical photographs of transplanted canine: initial eruption of transplanted canine four months after surgery (a); eight months after surgery the orthodontic bracket was debonded from crown of erupted canine (b and c); labial view of fully erupted canine and adjacent soft tissues at 1-year follow-up examination (d).

\section{Discussion}

The removal of ectopic lower canines necessitates osteotomy techniques and is usually very challenging. The extent of osteotomy depends on: severity of impaction, inclination, development and morphology of affected tooth and also on preferences and experience of the surgeon. Several techniques have been introduced to reduce extend of bone defects which are related to existence of ectopic tooth crypt within the bone but additionally are created during of a surgery while getting access to ectopic tooth. Fragmentation of ectopic canine is the treatment of choice in all orthodontic extraction cases. In patients with normal occlusal relations there are however contraindications for extraction of impacted or ectopic lower canines. Most of these cases can be treated with orthodontic exposure and attachment of orthodontic bracket followed by gradual orthodontic traction of impacted canine. In severe cases of impaction and migration (such as transmigration described in this case) orthodontic traction may however be not possible to perform or may pose a threat for roots of adjacent teeth. Surgical up righting (i.e. trans-alveolar auto transplantation) of canine to normal position may be alternative treatment option to save affected tooth in severe cases of ectopia.

Auto transplantation of the impacted tooth requires taking an enormous care to protect the hard and soft tissues of the root in order to decrease frequency and severity of complications after the surgery. The use of piezosurgery instruments can significantly reduce the extent and side effects of osteotomy associated with removal of the impacted tooth $[8,9]$. When auto transplantation of the tooth is performed, avoiding trauma to the periodontal tissues of the root becomes a priority. In the described case the trans-alveolar auto transplantation of ectopic canine was performed according to orthodontic plan. The application of orthodontic bracket and wire during the surgery allowed relatively easy removal of ectopic tooth from the bone. One year after surgery transplanted canine presented typical findings after transplantation of developing teeth such as: pulp obliteration, root growth, eruption and normal mobility. Two latter features resulted from normal healing of periodontal tissues after surgery and were related to minimally traumatic manner the tooth was treated during surgery. These features enhance normal alveolar bone development and for that reason are crucial in growing patients.

The orthodontic bracket with wire can be easily bonded to the enamel after exposure of the crown of impacted tooth. The application of controlled pulling forces during immediate removal of the impacted tooth from ectopic position reduces trauma to the periodontal ligament and cementum of the root. In this way vital structures of the root could be less damaged against the walls of the crypt within alveolar bone when compered to the conventional use of forceps, elevators or even periotomes. This may contribute to the better healing after surgery and may raise the number of successful transplantation. The bracket can be easily removed after healing and eruption of the tooth and it can replace with the standard bracket for later tooth alignment. Furthermore, this inexpensive technique may also complement other surgical procedures in reducing amount of osteotomy during extraction of the impacted teeth. It may limit the use of sharp or less precise instruments and decrease risk of injuries to roots of adjacent teeth, nerves and vessels.

\section{Conclusion}

The use of orthodontic bracket may contribute to reduce trauma to periodontal tissues during removal of impacted developing teeth for auto transplantation thus promoting uncomplicated healing after surgery.

\section{References}

1. Wertz RA (1994) Treatment of transmigrated mandibular canines. Am J Orthod Dentofacial Orthop 106(4): 419-427.

2. Andreasen JO (1997) the impacted mandibular canine. In: Andreasen JO, Petersen JK, Laskin DM (Eds.). Textbook and color atlas of tooth impactions. Diagnosis, treatment and prevention. Copenhagen: Munksgaard, pp. 168.

3. Joshi MR (2001) Transmigrant mandibular canines: a record of 28 cases and a retrospective review of the literature. Angle Orthod 71(1): 12-22.

4. Plakwicz P, Wojtaszek J, Zadurska M (2013) new bone formation at the site of auto transplanted developing mandibular canines: a case report. Int J Periodontics Restorative Dent 33(1): 13-20.

5. Kristerson L (1985) Auto transplantation of human premolars. A clinical and radiographic study of 100 teeth. Int J Oral Surg 14(2): 200-213.

6. Andreasen JO, Borum MK, Jacobsen HL, Andreasen FM (1995) Replantation of 400 avulsed permanent incisors. 4. Factors related to periodontal ligament healing. Endod Dent Traumatol 11(2): 76-89.

7. Czochrowska EM, Stenvik A, Bjercke B, Zachrisson BU (2002) Outcome of tooth transplantation: survival and success rates 17-41 years posttreatment. Am J Orthod Dentofacial Orthop 121(2): 110-119. 
8. Mantovani E, Arduino PG, Schierano G, Ferrero L, Gallesio G, et al. (2014) A split-mouth randomized clinical trial to evaluate the performance of piezosurgery compared with traditional technique in lower wisdom tooth removal. J Oral Maxillofac Surg 72(10): 1890-1897.
9. Nagori SA, Jose A, Bhutia O, Roychoudhury A (2014) Evaluating success of auto transplantation of embedded/impacted third molars harvested using piezosurgery: a pilot study. Acta Odontol Scand 72(8): 846-851.

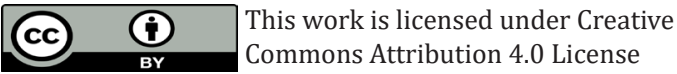

Submission Link: http://biomedres.us/submit-manuscript.php

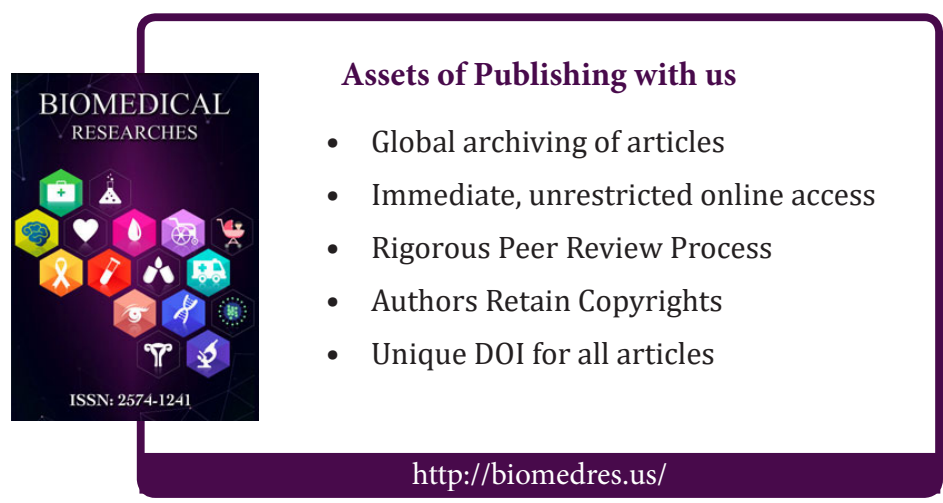

